\title{
The paperless partograph: can it be effective to replace the WHO modified partograph
}

\author{
Gitanjali Deka*, Rajashree Sharma, Gokul Chandra Das
}

Department of Obstetrics \& Gynaecology, Gauhati Medical College and Hospital, Guwahati, Assam, India

Received: 16 December 2015

Revised: 18 December 2015

Accepted: 08 January 2016

\section{*Correspondence:}

Dr. Gitanjali Deka,

E-mail: gitanjali.dk@gmail.com

Copyright: $(\odot$ the author(s), publisher and licensee Medip Academy. This is an open-access article distributed under the terms of the Creative Commons Attribution Non-Commercial License, which permits unrestricted non-commercial use, distribution, and reproduction in any medium, provided the original work is properly cited.

\begin{abstract}
Background: The partograph is a graphical representation of the various events of labour plotted against time. The main aim was to determine whether the paperless partograph can replace the WHO partograph to monitor labour and aid in decision making.

Methods: It is a hospital based prospective analytical study. The course of labour in 400 women with term singleton uncomplicated pregnancies were studied by using either partographs. 12 resident doctors were included to assess the user friendliness and asked to fill 240 separate partographs (120 each of WHO Modified and Paperless partograph).

Results: The maternal and perinatal outcome was comparable between both the partographs. The Paperless partograph was however more user-friendly than the WHO partograph $(\mathrm{p}<0.0001)$.

Conclusions: The Paperless partograph was found to be as effective as the WHO partograph in the effective management of labour. It is more user-friendly and has promising prospects to replace the WHO partograph.
\end{abstract}

Keywords: Decision making, Prospective studies, Monitoring, Labor

\section{INTRODUCTION}

The partograph is a graphical representation of the various events of labour plotted against time. It serves to be a very cost effective and affordable health intervention for monitoring labour and appropriate decision making. The partograph was originally developed by Friedman in 1954 based on his observations on labours of women in Zimbabwe (then Rhodesia). It was later modified by Philpott and Castle by inclusion of the alert and action lines. ${ }^{1}$ These partographs formed the basis of the WHO partograph which was universally adopted for monitoring labour at the Safe Motherhood Conference. ${ }^{2}$ The earliest WHO partograph was the Composite partograph which was further modified in 2000 by eliminating the latent phase to yield the WHO modified partograph.
However the current problem is that the use and complete documentation of the Partograph is notoriously low in low resource countries. Several factors have been implicated to the cause of this low use such as lack of awareness and proper training, low availability of partographs, negative perceptions of the partograph, high patient load, inadequate staff at the facilities, lack of supervision, and negative attitudes among some of the health workers. ${ }^{3}$ Continuous monitoring of labour and provision of rapid care to deal with problems are most crucial for preventing adverse obstetric outcomes related to childbirth. Debdas argues that the WHO partograph fails to meet the present requirements and proposes the Paperless partograph. The Paperless partograph is a simple, graph less, non-time consuming method which only involves the calculation of an expected time of 
delivery (ETD). It identifies slow progress of labour, the time to intervene and terminate labour or to transfer a woman to a higher centre with facilities for caesarean section. $^{4}$

Hence the present study was intended to determine whether the Paperless partograph replace the WHO Modified partograph in the effective management of labour.

\section{METHODS}

The present study was carried out in the Department of Obstetrics and Gynaecology, Gauhati Medical College \& Hospital (GMCH), Assam, India from 01 May 2014 to 30 April 2015 after obtaining the clearance from the institutional ethical committee.

It was a hospital based prospective analytical study. During the period of our study we studied the course of labour in 400 women with singleton, term (37-40 weeks) pregnancies with vertex presentation in spontaneous labour without any complications attending the labour room for delivery. Detailed bio-data and history of all patients were taken and those willing to participate after an informed and written consent were included in the study.

Half of the patients (200) were monitored by the WHO Modified partograph in group A and the other half by Paperless partograph in group B. The plotting of the partographs was started as soon the cervical dilatation was $4 \mathrm{~cm}$ along with regular painful uterine contractions. The outcome of labour was recorded at the end of each partograph.

For determining user friendliness 240 separate partographs (120 WHO Modified and 120 Paperless) were given to 12 resident doctors working alternately in shift duties. They were instructed on the use of either partographs. They randomly used these partographs for monitoring labour and based on their personal experience gave scores to each of the partographs on the basis of; (a) user friendliness (1-10); (b) teachability (1-10); and (c) overall usefulness (1-10). All partographs were checked for completeness.

All the results were plotted in a master chart and analysis was made by using the Chi-square test and $p$ value $<0.05$ was considered significant.

\section{RESULTS}

During the study period 400 women in labour were monitored by the WHO and paperless partograph (200 each). The baseline characteristics of the patients are as mentioned below (Table 1).

In our study it was observed that most of the cases delivered before reaching the alert line/ETD i.e $77 \%$ in case of WHO partograph and $83 \%(\mathrm{p}=0.13) .18 .5 \%$ of cases monitored by WHO partograph and $15 \%$ of cases monitored by Paperless partograph delivered between the alert line/alert ETD and action line/action ETD $(p=0.3)$. A minor proportion $4.5 \%$ in case of WHO partograph and $2 \%$ in case of Paperless partograph delivered beyond the action line/action ETD $(\mathrm{p}=0.15)$. These cases were properly reassessed and delivered so that none of the cases were complicated by prolonged labour and its sequel (Table 2).

Table 1: Baseline characteristics of the participants.

\begin{tabular}{|llll|}
\hline & Range & \multicolumn{2}{c|}{ Gean \pm SD } \\
\hline Age (years) & $17-36$ & $26 \pm 3.6$ & $25 \pm 3.2$ \\
\hline $\begin{array}{l}\text { Nutritional } \\
\text { status } \\
\text { (BMI Kg/m }{ }^{2} \text { ) }\end{array}$ & $16-31$ & $24.17 \pm 3.6$ & $23.4 \pm 3.4$ \\
\hline $\begin{array}{l}\text { Gestational age } \\
\text { (weeks) }\end{array}$ & $37-41$ & $37.6 \pm 1.04$ & $37.7 \pm 0.78$ \\
\hline $\begin{array}{l}\text { Temperature } \\
\text { (degree/Celsius) }\end{array}$ & $36-39$ & $36.9 \pm 0.2$ & $36.7 \pm 0.3$ \\
\hline $\begin{array}{l}\text { Pulse } \\
\text { (beats /min) }\end{array}$ & $60-90$ & $72.5 \pm 4.48$ & $72.6 \pm 3.73$ \\
\hline $\begin{array}{l}\text { Systolic BP } \\
\text { (mm Hg) }\end{array}$ & $90-120$ & $108.5 \pm 9.5$ & $107.5 \pm 8.9$ \\
\hline $\begin{array}{l}\text { Diastolic BP } \\
\text { (mm Hg) }\end{array}$ & $60-90$ & $75.9 \pm 9.2$ & $77.1 \pm 8.9$ \\
\hline $\begin{array}{l}\text { Uterine } \\
\text { contractions/10 } \\
\text { mins }\end{array}$ & $1-5$ & $2.7 \pm 1.06$ & $2.74 \pm 1.16$ \\
\hline
\end{tabular}

Table 2: Distribution of cases in relation to alert and action line/ETD.

\begin{tabular}{|llll|}
\hline Variable & $\begin{array}{l}\text { Group A } \\
(\%)\end{array}$ & $\begin{array}{l}\text { Group B } \\
(\%)\end{array}$ & $\begin{array}{l}\text { P } \\
\text { value }\end{array}$ \\
\hline $\begin{array}{l}\text { Within Alert } \\
\text { line/Alert ETD }\end{array}$ & $\begin{array}{l}154 \\
(77 \%)\end{array}$ & $\begin{array}{l}166 \\
(83 \%)\end{array}$ & 0.13 \\
\hline $\begin{array}{l}\text { Between Alert line } \\
\text { /Alert ETD and } \\
\begin{array}{l}\text { Action line/ Action } \\
\text { ETD }\end{array}\end{array}$ & 37 & 30 & 0.3 \\
\hline $\begin{array}{l}\text { Beyond Action } \\
\text { line/ETD }\end{array}$ & 9 & $(18.5 \%)$ & \\
\hline Total & $(4.5 \%)$ & $(2 \%)$ & 0.15 \\
\hline
\end{tabular}

$88.5 \%$ of cases monitored by the Paperless partograph and $85 \%$ cases monitored by WHO partograph had a spontaneous delivery. Caesarean section was required in only $6 \%$ cases monitored by Paperless partograph as against $10.5 \%$ cases of WHO partograph $(\mathrm{p}=0.18)$. Almost the same number of cases, 11 in Paperless group and 9 in WHO group required assisted delivery. These results were not statistically significant (Table 3 ). 
Table 3: Mode of delivery.

\begin{tabular}{|lrrrrr|}
\hline $\begin{array}{l}\text { Variable } \\
\text { Spontaneous }\end{array}$ & Group A & \multicolumn{2}{c|}{ Group B } & P value \\
$\begin{array}{l}\text { vaginal } \\
\text { vasisted }\end{array}$ & 170 & $85 \%$ & 177 & $88.5 \%$ & 0.3 \\
\hline $\begin{array}{l}\text { Assinal } \\
\text { vaginal } \\
\text { (instrumental ) }\end{array}$ & 9 & $4.5 \%$ & 11 & $5.5 \%$ & 0.18 \\
\hline $\begin{array}{l}\text { Caesarean } \\
\text { section }\end{array}$ & 21 & $10.5 \%$ & 12 & $6 \%$ & 0.1 \\
\hline
\end{tabular}

On analysing the perinatal outcome we found that the average APGAR score after 1 min was $8.1 \pm 1.7$ in group $A$ and $8.3 \pm 0.7$ in group $B$ respectively $(p=0.12)$. The Apgar score after 5 mins had an average of 9.6 \pm 0.7 in cases randomized to the WHO partograph and 9.45 \pm 1.6 in those subjected to the Paperless one. Thus similar perinatal outcome was seen in both groups which had no statistically significant difference (Table 4).

Table 4: Perinatal outcome.

\begin{tabular}{|lccl|}
\hline $\begin{array}{l}\text { Variable } \\
(\text { Mean } \pm \text { SD ) }\end{array}$ & Group A & Group B & P value \\
\hline $\begin{array}{l}\text { Neonatal weight } \\
(\mathrm{kg})\end{array}$ & $2.7 \pm 0.41$ & $2.7 \pm 0.38$ & 0.44 \\
\hline $\begin{array}{l}\text { APGAR score at } \\
1 \text { min }\end{array}$ & $8.1 \pm 1.7$ & $8.3 \pm 0.7$ & 0.12 \\
\hline $\begin{array}{l}\text { APGAR score at } \\
5 \text { min }\end{array}$ & $9.6 \pm 0.7$ & $9.45 \pm 1.6$ & 0.2 \\
\hline
\end{tabular}

The resident doctors scored the two partographs for each of the following categories: user friendliness, teachability and overall usefulness. The mean user friendliness score

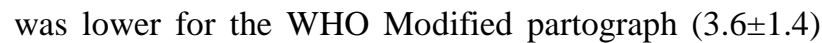
while it was high for the paperless partograph $(7.9 \pm 0.65)$ which was statistically significant with $\mathrm{p}<0.0001$. Most of the resident doctors $(66.6 \%)$ preferred to use the paperless partograph rather than the WHO partograph $(33.4 \%)$ as it was simple graphless and less time consuming (Table 5).

Table 5: User-friendliness.

\begin{tabular}{|lccc|}
\hline Variable & Group A & Group B & P value \\
\hline User friendliness & $3.6 \pm 0.8$ & $7.9 \pm 0.65$ & $<0.0001$ \\
\hline Teachability & $3.6 \pm 1.4$ & $8.08 \pm 0.9$ & $<0.0001$ \\
\hline Overall usefulness & $7.6 \pm 0.4$ & $7.75 \pm 0.45$ & 0.39 \\
\hline
\end{tabular}

\section{DISCUSSION}

Continuous monitoring of labour along with an agreed protocol for management of labour are key factors for adequate intrapartum care. The WHO Modified partographs is an excellent means to monitor labour and aid in appropriate decision making. It serves to be a "warning system" for early identification of abnormal labour particularly in low resource countries. However currently it is seen that the WHO Modified partograph is neither used nor properly documented which has affected the quality of intrapartum care.

In this context the "Paperless Partograph" proposed by Debdas serves to be an easier method for monitoring labour. The present study was conducted at the tertiary centre of Gauhati Medical College and Hospital to determine whether the paperless partograph can be used to replace the WHO partograph.

In the present study we found that the Paperless partograph was as efficient as the WHO partograph for monitoring labour. Most of the cases (83\%) monitored by the Paperless had a normal course of labour and delivered before the alert ETD which was similar to those monitored by the WHO partograph (77\%). Only 34 $(17 \%)$ cases monitored by paperless partograph crossed the alert ETD out of which $2 \%$ cases delivered beyond the action ETD. Similarly $46(23 \%)$ cases crossed the alert line in the WHO group out of which only $9(4.5 \%)$ cases delivered beyond the action line. Almost similar results were seen in a study conducted by Deblina et al in West Bengal observed that $14.5 \%$ cases monitored by the paperless partograph delivered between alert and action ETD and only $1.8 \%$ beyond the action ETD. ${ }^{5}$

Another study conducted with the Paperless partograph in Odisha by Dr. Prakash et al found that $75.5 \%$ of primigravida and $90.7 \%$ of multigravida delivered before the alert ETD. ${ }^{6}$

The success rate of spontaneous deliveries in our study was $88.5 \%$ in case of the Paperless partograph and $85 \%$ in the WHO group. Similar results were also observed by Lingegowda $\mathrm{K}$ in his study on comparison between WHO and paperless partograph conducted in PESIMSR, Kuppam where $44 \%$ cases monitored by WHO partograph and $74 \%$ cases monitored by paperless partograph had a spontaneous delivery. ${ }^{7}$

The rate of caesarean section was $6 \%$ of cases of paperless group and $10.5 \%$ in cases monitored by WHO partograph. A study by Agarwal k et al in Uttar Pradesh observed a rate of $1 \%$ caesarean sections with the Paperless partograph. ${ }^{8}$

Another study conducted by Fatouh E et al in Egypt from 1st March to last of August 2014 observed a CS rate of $23.1 \%$ with the Paperless partograph. ${ }^{9}$ Thus the paperless partograph was found similar and effective in monitoring labour as compared to the WHO partograph.

There was no significant statistical difference in the neonatal outcome of these parturients in our study. The average Apgar score at $1 \mathrm{~min}$ was $8.3 \pm 0.7$ in cases monitored by paperless partograph and $8.1 \pm 1.7$ in cases monitored by WHO partograph. The average Apgar score after 5 mins was $9.45 \pm 1.6$ in paperless group and 9.6 \pm 0.7 in WHO group. Prompt resuscitative measures were 
instituted so that there was no reported neonatal mortality in our study.

Most of the resident doctors $(66.6 \%)$ preferred to use the paperless partograph rather than the WHO partograph (33.4\%) for monitoring. Similar results were seen by Fatouh E et al where more than three quarter $(75 \%)$ of nurses preferred to use the paperless partograph over the traditional partograph.

In the present study it was seen that the paperless partograph was as efficient as the WHO partograph in monitoring labour and appropriate decision making. The perinatal outcome was also comparable in both the groups. However the Paperless partograph was found more user friendly than the WHO partograph and was more preferred by the resident staff. We recommend further studies with the paperless partograph even at the grass root levels of health providers such as skilled birth attendants in order to test its efficacy at the community level.

\section{CONCLUSIONS}

The total number of deliveries in Gauhati Medical College and Hospital during the study period was 22,500 which have a great workload on the attending staff. In our study we found that the paperless partograph was as efficient as the WHO partograph in monitoring labour and to decide further management. However the paperless partograph was easier to maintain and more user-friendly and hence could be easily plotted even by those with minimal formal training on it. Thus it can serve to replace the WHO partograph particularly in areas with high workload of patients and shortage of manpower.

\section{Funding: No funding sources}

Conflict of interest: None declared

Ethical approval: The study was approved by the Institutional Ethics Committee

\section{REFERENCES}

1. Freidman EA. Primigravidae Labor; A graphicostatistical analysis. Obstetrics and Gynaecology. 1955;6;567-89.

2. Surveys in Bayelsa State; 2006. WHO: Beyond the Number. Reviewing Maternal Deaths and Complications to Make Pregnancy Safer: WHO, Geneva, 2004.

3. Ogwang S, Karyabakabo Z, Rutebemberwa E. Assessment of partogram use during labour in Rujumbura Health Sub District, Rukungiri District, Uganda. African Health Sciences. 2009;9(Supplement 1):S27-34.

4. Debdas AK. Paperless Partogram. 41st Annual Scientific Session 2008; Sri Lanka college of Obstetrics and Gynaecologists. SLJOG. 2008;30(1):124.

5. Roy D, Dey R. ETD- Expected Time Of Delivery- A New Simple Clinical Tool For Management Of Labour. AICOG Patna. 2014:2.

6. Mishra P, Nayak L. ETD-Expected Time of Delivery- A New Simple Clinical Tool for Management of Labour. AICOG Chennai.2014.

7. Lingegowda K, Bhuvaneswari, Shailaja N, Kulkarni $\mathrm{N}$, Bhatt SB, Vimala. Comparison of WHO partograph with Paperless partograph in the management of labour and to determine which is more use friendly"; AICOG Patna. 2014:106-7.

8. Agarwal K, Agarwal L, Agarwal VK, Agarwal A, Sharma M. Evaluation of Paperless Partogram as a Bedside Tool in the Management of Labor. Journal of Family Medicine and Primary Care. 2013;2(1):479.

9. Fatouh E, Ramadan S. Effect of using Paperless Partogram on the Management and Outcome of Labour and the Nurses' Opinion. Journal of Education and Practice. 2015;6(8):17-23.

Cite this article as: Deka G, Sharma R, Das GC. The paperless partograph-can it be effective to replace the WHO modified partograph. Int J Reprod Contracept Obstet Gynecol 2016;5:452-5. 